Nisho-96/4

\title{
Ferromagnetic Domain Wall and Primordial Magnetic Field
}

\author{
Aiichi Iwazaki \\ Department of Physics, Nishogakusha University, Shonan Ohi Chiba 277, Japan.
}

(August 7, 1996)

\begin{abstract}
We show that coherent magnetic field is generated spontaneously when a large domain wall is created in the early universe. It is caused by two dimensional massless fermions bounded to the domain wall soliton. We point out that the magnetic field is a candidate of primordial magnetic field 98.80.Cq, 95.85.Sz, 11.15.Tk, 11.30.Er
\end{abstract}

Typeset using REVTEX 
Domain walls [1] arise in any unification models with a discrete symmetry in Higgs potentials and the symmetry is often used to reduce complication in the potentials. Unfortunately the domain walls are unfavorable in cosmological point of view [2]; energy density of the domain wall dominates in the universe and invalidates the scenario of the history of the early universe. To cure this problem several ideas [2 [5] are proposed, but there is not yet a definite solution. Here we do not address this problem although we suggest a possible solution for the problem throughout analysis of new features of the domain wall.

In this paper we analyze magnetic properties of the domain wall ( hereafter we simply call it as the wall ) and derive a favorable consequence of an origin of primordial magnetic field [6] in the universe. We show spontaneous generation of a magnetic field in the presence of the wall. A size of the coherence of the magnetic field is comparable to that of the wall. ( We assume the existence of a domain wall with its size $L$ being the same as the distance to the horizon, as indicated by numerical simulation [7] of the creation of the walls in the early universe ). The essence of the result is in the presence of fermion zero modes [8] on the wall; the zero modes exist in general when fermion couples with the domain wall soliton. It implies the existence of massless fermions on the two dimensional wall, although the fermions are massive when they are unbounded to the wall. Such two dimensional massless fermions have a specific magnetic property when they have electric charges; their magnetic moments are infinite ( we may define the magnetic moment such as a derivative of fermion energy in terms of magnetic field evaluated at the value of the vanishing field ). This fact suggests that the energy of the fermion gas ( two dimensional fermion gas confined to the wall ) may has unusual dependence of magnetic field $B$ perpendicular to the wall; usually the energy is proportional to $B^{2}$. As we will show later, the free energy of the fermion gas is proportional to $B$. The sign of the coefficient of this term is negative. Thus the amount of the decrease of the energy is proportional to $B$. On the other hand the field energy is proportional to $B^{2}$. Therefore, spontaneous generation of magnetic field can occur since total energy of the system decreases with the presence of the sufficiently weak magnetic field. As the size of the wall is the same as the distance to the horizon, the scale of the coherence 
of such magnetic field is so large that it may be a candidate of primordial magnetic field leading to intergalactic or galactic magnetic field [9]. The strength of the field depends on the periods when the wall is created and when it disappears. For instance, if it is created in the electroweak phase transition at about the temperature of $1 \mathrm{TeV}$, the strength of the field generated at the temperature is about $10^{9}$ Gauss. It leads to the field with its strength $10^{-15}$ Gauss at recombination of photons and electrons. We suggest that since the generation of the magnetic field breaks explicitly CP invariance, it might lead to a possible solution for the domain wall problem in a model with spontaneous $\mathrm{CP}$ violation.

We now present detail calculations. Hereafter we consider a large flat domain wall with its size $L$ assumed to be the same as the distance to the horizon. First we discuss fermionic zero modes [8]. The modes arise in general when the fermion $\psi$ couples with a Higgs field $\phi$; strictly speaking the existence of the modes depends on how the field $\phi$ couples with the fermion. Here we assume for simplicity the real Higgs field $\phi$ whose potential has a discrete symmetry, $\phi \rightarrow-\phi$ and the following Yukawa coupling, $g \bar{\psi} \psi \phi+h . c$. . The field $\phi$ behaves near the wall supposed to be sited at $x_{3}=0$ such as $\phi\left(x_{3}\right) \rightarrow \pm v$ as $x_{3} \rightarrow \pm \infty$; $\pm v$ are vacuum expectation values of the Higgs field $\phi$. The wall is extending in $x_{1}$ and $x_{2}$ directions. Then, it is easy to find the zero modes by solving a Dirac equation with energy $E$ and derivatives $\vec{\partial}$ transverse to the wall put to be zero, $\left(E \gamma_{0}+i \vec{\gamma} \vec{\partial}+i \gamma_{3} \partial_{3}+g \phi\right) \psi=0$ where $\vec{\gamma}(\vec{\partial})$ is a two dimensional vector. Adopting the representation of gamma metrics,

$$
\gamma_{0}=\left(\begin{array}{cc}
1 & 0 \\
0 & -1
\end{array}\right) \text { and } \gamma^{i}=\left(\begin{array}{cc}
0 & \sigma_{i} \\
-\sigma_{i} & 0
\end{array}\right)
$$

we find the solutions with zero energy,

$$
\psi=\left(\begin{array}{c}
u \\
i \sigma_{3} u
\end{array}\right), \quad u=\left(\begin{array}{l}
a \\
b
\end{array}\right) \exp \left(-z\left(x_{3}\right)\right)
$$

where $z\left(x_{3}\right)$ is defined as $\partial z\left(x_{3}\right) / \partial x_{3}=\phi\left(x_{3}\right) ; a$ and $b$ are constants. Thus there are two zero energy modes. These may be viewed as zero energy bound states of massive fermion $\psi$ with its mass $m=\sqrt{g} v$. It turns out that when the states move in transverse directions on the wall ( in this case $a$ and $b$ are functions of $x_{1}$ and $x_{2}$ ), their energies are proportional to their 
momenta. Thus they are massless fermions in two dimensional space of the wall. Note that such states attached to the wall loose 2 dynamical degrees of freedom; components $u_{1}$ and $u_{2}$ of $\psi=\left(\begin{array}{l}u_{1} \\ u_{2}\end{array}\right)$ are not independent with each others. Namely spin degrees of freedom are lost so that the two dimensional massless fermion ( anti-fermion ) has only one dynamical degree of freedom [10].

In order to show spontaneous generation of magnetic field we calculate a thermodynamical potential and a zero point energy of these fermions under a magnetic field perpendicular to the wall. The fermions are supposed to have electric charge, e. For the purpose we need to find energy spectrum under a constant magnetic field $B ; \vec{B}=(0,0, B)$. Choosing a gauge such as $\vec{A}=\left(-x_{2}, x_{1}, 0\right) B / 2$, we solve the above Dirac equation with gauge potential $\vec{A}$. Then, relevant solutions take the form in eq(2) with both of a and b being functions of $x_{1}$ and $x_{2}$; these are solutions of the cyclotron motions of the fermions bounded to the wall. Their spectra can be obtained easily, $E_{n}= \pm \sqrt{2 e B n}(n \geq 0)$ with degeneracy per unit erea being given by $e B / 2 \pi$. Eigenstates are characterized with integer $n$ and orbital angular momentum $m(n, m \geq 0)$

$$
\psi=\left(\begin{array}{c}
u \\
i \sigma_{3} u
\end{array}\right), \quad u=c\left(\begin{array}{l}
v_{1} \\
v_{2}
\end{array}\right)
$$

with

$$
v_{1}=\rho^{m} e^{i m \theta} L_{m}{ }^{(n)} e^{-e B \rho^{2} / 4}, \quad \text { and }, \quad v_{2}=\rho^{m} e^{i(m+1) \theta} \partial_{\rho} L_{m}{ }^{(n)} e^{-e B \rho^{2} / 4} / E_{n}
$$

where $L_{m}{ }^{(n)}\left(e B \rho^{2} / 2\right)$ is Laguerre function and $c$ is a normalization constant $\left(\rho^{2}=x_{1}^{2}+x_{2}^{2}\right.$ ) .

It should be mentioned that these states representing fermions bounded to the wall need to satisfy a energy condition such as $E_{n} \leq m$. Otherwise, the states represent fermions unbounded to the wall and hence are not relevant to the property of the wall. This energy condition is distinctive of the fermions on the wall. Besides those solutions, there are solutions describing states of cyclotron motions in $x_{1}-x_{2}$ plane and scattering states in $x_{3}$ direction. These are also not relevant for intrinsic properties of the wall. 
Strictly speaking, there are energy eigenstates localized on the wall with their energies higher than m. But it seems that these states are unstable against any couplings with scattering states with the same energies. They decay and corresponding fermions go away from the wall. Hence it is conceived that they are irrelevant for the property of the wall.

Now, let us calculate thermodynamical potential $\Omega$ under the condition that the temperature is much smaller than the mass of the fermion. Then it is given for free gas of fermions such that

$$
\Omega=\Omega_{+}+\Omega_{-}+\Omega_{0}, \quad \Omega_{ \pm}=-\beta^{-1} N_{d} \sum_{n=1}^{\infty} \log \left(1+e^{-E_{n} \beta}\right), \quad \Omega_{0}=-\beta^{-1} N_{d} \log 2
$$

where $N_{d}=e B L^{2} / 2 \pi$ is degeneracy of a Landau level ( $L^{2}$ is surface area of the wall ). Summation $\sum_{E_{n} \leq m}$ resulted from the energy condition has been replaced by the summation $\sum_{n=1}^{\infty}$ owing to the condition, $\beta^{-1}<<m$. The indices $( \pm, 0)$ denote quantities of fermions, anti-fermions and zero energy states $\left(E_{n=0}=0\right)$, respectively. $\beta$ is inverse temperature. For simplicity we have assumed a vanishing chemical potential associated with the fermion number, namely the fermion number in the universe is assumed to be negligibly small just as baryon number in the universe.

As we are interested in the behavior of the system at small magnetic field, the sum over integer $n$ can be evaluated by using Poisson resummation formula,

$$
\sum_{n=1}^{\infty} f\left(n \hat{\beta}^{2}\right)=\sum_{m=-\infty}^{\infty} \int_{0}^{\infty} d x f\left(x \hat{\beta}^{2}\right) e^{2 \pi i m x}=\int_{0}^{\infty} d x f\left(x \hat{\beta}^{2}\right)+2 \sum_{m=1}^{\infty} \int_{0}^{\infty} d x f\left(x \hat{\beta}^{2}\right) \cos (2 \pi m x)
$$

with $\hat{\beta}^{2} \equiv 2 e B \beta^{2}$, where $f(x)=\log \left(1+e^{-\sqrt{x}}\right)$. Expanding the second term in small $\hat{\beta}$, we obtain the thermodynamical potential for $\hat{\beta} \rightarrow 0$ such that

$$
\Omega=-\frac{1}{2 \pi} \beta^{-3} L^{2} \int_{0}^{\infty} \frac{y^{2} d y}{1+e^{y}}+\frac{\sqrt{2 e B} e B \zeta(-1 / 2) L^{2}}{2 \pi}+O\left(\beta(e B)^{2}\right)
$$

where the first term represents a contribution of free massless fermions without the magnetic field and the second one represents a correction by the weak magnetic field $e B<<\beta^{-2}$. 
This first term can be also obtained by using the free fermion energy, $E_{k}=|k|$ ( $k$ is 2 dimensional momentum ) and by taking account of both of fermion and anti-fermion with only one dynamical degree of freedom.

Similarly we calculate a zero point energy of the two dimensional massless fermion on the wall. In the case we need to take care the energy condition; energies of these fermions are restricted such that $E_{n} \leq m$. This is because the fermions with higher energies than their three dimensional mass $m=g v$ are not bounded to the domain wall. Thus zero point oscillations of such fermions are not associated with the property of the wall, but with the property of the outside of the wall. Therefore the zero point energy of the wall is given by

$$
E_{0}=-\sum_{n=1}^{F} \sqrt{2 e B n} N_{d} \approx-\frac{m^{3} L^{2}}{6 \pi}-\frac{e B m L^{2}}{4 \pi}-\frac{\sqrt{2 e B} e B \zeta(-1 / 2) L^{2}}{2 \pi}
$$

where $F=m^{2} / 2 e B$ is the number of Landau levels, energies of whose states are less than the mass $m$ of the fermion; $\zeta(z)$ is zeta-function. We have taken a limit of small magnetic field ( $e B<<m^{2}$ ) in the calculation. The first term represents a zero point energy of the fermion without magnetic field. The term can be also expressed by the integration, $-L^{2} \int_{|k|<m}|k| d k^{2} /(2 \pi)^{2}$. This quantity should be renormalized to surface energy of the wall. The third term has already been obtained [11] as a zero point energy of two dimensional massless fermion without the energy condition mentioned above.

Comment is in order. In the calculation of the zero point energy we have included only effects of localized states whose energies are less than $m$. Namely, we have not taken into account of the effects of states localized on the wall with higher energies than $m$. Such states really exist in the case of no their coupings with the scattering states. The states, however, are quite unstable against the coupings; these localized states decay and disappear. Hence it is reasonable to conceive that the only states which really exist are scattering states with energies higher than $m$ and bound states with energies less than $m$. Thus we expect physically that in a realistic circumstance we may neglect the effects of the localized states with higher energies than $m$. On this point we elaborate it in future publication.

We can see that the magnetic field reduces both the thermal energy of the fermion gas 
and the zero point energy; the amounts of the reduction are proportional to $B \sqrt{B} L^{2}$ and $B L^{2}$ respectively. This fact leads to spontaneous generation of magnetic field. Namely when the magnetic field is present in the universe the whole free energy $E_{B}$ associated with the magnetic field is given by the above energies of two dimensional fermion gas and the energy of the magnetic field itself,

$$
E_{B}=-\frac{e B m L^{2}}{4 \pi}+\frac{B^{2} L^{3}}{2 \mu}
$$

where $L^{3}$ is the volume inside the horizon. $\mu$ is permeability of the universe. ( The thermal effect of the order of $B^{3 / 2}$ in eq(可) cancels with the corresponding effect of the vacuum energy [12]. But this cancellation does not necessarily hold in any cases: For instance when the chemical potencial of the fermion in the universe is nonvanishing, the cancellation does not occur. In the case the thermal effect dominates over the effect of the vacuum energy.) Hence, the magnetic field generated spontaneously is obtained by minimizing this energy,

$$
B_{r}=\frac{e m \mu}{4 \pi L}
$$

This is the magnetic field associated with the domain wall soliton in the universe. The strength of the field becomes small with the expansion of the universe. In the radiationdominated universe the distance $L$ to the horizon behaves with temperature $\beta^{-1}$ such as $f M_{p l} \beta^{2}$ ( $M_{p l}$ is the Planck mass and $f$ is the total number of massless degrees of freedom at temperature $\beta^{-1}$ ) so that $B_{r}$ decreases in such a way as $B_{r} \sim \beta^{-2} / f M_{p l}$. Numerically, when the domain wall arises at the electroweak phase transition, e.g. $\beta^{-1} \sim 100 \mathrm{GeV}$ ( $f \sim 100$ ), the magnitude of the magnetic field is of order of $10^{6}$ Gauss with the use of the top quark mass $m=175 \mathrm{GeV}$.

Here we wish to understand a mechanism of the above phenomena ( ferromagnetism ) of the wall. For the purpose we calculate magnetization, $M=-\partial E_{B} /\left.\partial B\right|_{B \rightarrow 0}=e m L^{2} / 4 \pi$. It turns out that the magnetization originates in the zero point energy. Namely, each state with negative energy $E_{n}^{\prime}=-\sqrt{2 e B n}$ contributes a positively infinite magnetic moment, while the number $N_{d}\left(=e B L^{2} / 2 \pi\right)$ of the states with the energy vanishes as $B \rightarrow 0$ and 
the number $F\left(=m^{2} / 2 e B\right)$ of the Landau levels in the Dirac sea becomes infinite as $B \rightarrow 0$. These effects leads to the spontaneous generation of the magnetic moment of the wall. This is a mechanism leading to the ferromagnetism of the wall in the case of the sufficiently small temperature.

We have so far assumed in the evaluation of the thermodynamical potential that the temperature is much smaller than the mass of the fermion, i.e. $\beta^{-1}<<m$. The fermions bounded to the wall never escape even if they are excited thermally: So we have performed infinite sum in the previous calculation. On the contrary when the temperature is much larger than the mass of the fermion, they can escape from the wall. In the evaluation of the thermodynamical potential in the case we should take account of the energy condition; only the fermions with their energies less than the mass $m$ contribute to the potential,

$$
\Omega_{ \pm,}=-\beta^{-1} N_{d} \sum_{n=1}^{F} \log \left(1+e^{-E_{n} \beta}\right) \cong-\beta^{-1} N_{d} F \log 2, \quad \Omega_{0}=-\beta^{-1} N_{d} \log 2
$$

where we have taken the temperature $\beta^{-1}$ being much larger than the mass $m$ of the fermion. Therefore the whole free energy depending on the magnetic field is

$$
E_{B}=-\frac{e B L^{2} \log 2}{2 \pi \beta}+\frac{B^{2} L^{3}}{2 \mu}
$$

where the zero point energy has been neglected because $\beta^{-1}$ is much larger than $m$ so that the energy is much smaller than the thermal energy of the first term in eq(12). The thermal energy dominates over the zero point energy. We see that imposition of the magnetic field reduces the free energy of the fermion gas. Then the field generated spontaneously is given by

$$
B_{r}=\frac{e \mu \log 2}{2 \pi \beta L}
$$

Numerically, $B_{r} \cong 10^{6}$ Gauss at $\beta^{-1}=100 \mathrm{GeV}$, or $B_{r} \cong 1$ Gauss at $\beta^{-1}=1 \mathrm{GeV}$.

Comparing this result with the previous one in eq(10), we understand that the magnetic field $B_{r}$ originates in the zero point oscillation of the fermion when the temperature is less than the mass of the fermion, while the field originates in the thermal effects of the fermion 
when the temperature is higher than the mass. In the latter case the magnetic field increases the number $N_{B}$ of the states with energies less than the mass $m$,

$$
N_{B}-N_{B=0}=\frac{e B L^{2}}{2 \pi} \sum_{n=0}^{F-1}-\frac{L^{2}}{(2 \pi)^{2}} \int_{|k| \leq m} d k^{2}=\frac{e B L^{2}\left(F-m^{2} / 2 e B\right)}{2 \pi}>0
$$

where $F$ is such that $\sqrt{2 e B F}>m>\sqrt{2 e B(F-1)}$. Thus it leads to increases entropy $S$ of the gas and hence decreases the free energy $\left(=E-S \beta^{-1} \approx-S \beta^{-1}\right)$. This is the reason of the spontaneous generation of the magnetic field when the temperature is higher than the mass of the fermion $\left(E \approx m L^{2}<<S \beta^{-1}\right)$.

As we have shown, the spontaneous generation of magnetic field occurs in the presence of the domain wall soliton. The origin of the magnetic field is a natural consequence of fermion dynamics on the domain wall soliton. Hereafter we wish to discuss briefly phenomenological application of the result.

Let us consider a realistic model where the wall is created at the electroweak phase transition and the fermions are quarks or lepton: Such models exist, e.g. a next to minimal supersymmetric standard model [13]. The transition temperature may be about $1 \mathrm{TeV}$ and it is much larger than any masses of quarks and leptons. Thus the above formula in eq(13) is applicable for this case. Then, the magnetic field is roughly $10^{9}$ Gauss at the temperature of $1 \mathrm{TeV}$ with its coherent length $L=10^{-2} \mathrm{~cm}$. This magnetic field is naively expected to lead to a magnetic field of $10^{-15}$ Gauss with coherent length $10^{10} \mathrm{~cm}$ around the period of the recombination. Note that the evolution of the field is such that $B a^{2}=$ constant because its flux is conserved owing to very large electric conductivity of the universe [9]; $a$ is the cosmic scale factor in Robertson-Walker metric. As the universe expands, the size of the wall becomes large and the magnetic field becomes weak. But, we expect that a mechanism [2 50 begins to work for destabilizing the wall before the wall dominates the energy density of the universe. Then the wall decays with its shape topologically equivalent to sphere. In the case the strength of the magnetic field is turned out to be proportional to the inverse of radius of the sphere. Hence the magnetic field becomes stronger as the spherical wall shrinks more. In these processes of the wall evolution the magnetic fields are printed in medium 
of the universe because their fluxes are conserved. Hence the magnetic fields with various strengths and sizes of coherence are left in the evolution of the wall. These are candidates of primordial magnetic fields leading to galactic or stellar magnetic field. To determine the strength ( the coherent length ) of the field more precisely, we have to include dissipation of magnetic field and the number of the fermion species, and to know when and how the wall disappears.

Finally we point out a possible solution for the domain wall problem in a electroweak model with spontaneous CP violation. In the model domain walls may arise associated with the spontaneous breakdown of this discrete CP invariance. If quarks or leptons couple with Higgs fields of the wall and give rise to zero modes, then the magnetic field may be generated spontaneously in a similar way as we have discussed. This field is not CP invariant. Thus an effective Higgs potential may involve a CP non invariant term which would be generated by fermion loops with the effect of this magnetic field. The term induces a vacuum pressure [2] which makes eventually the wall to disappear before it dominates in the universe. A problem in this scenario is whether or not this CP non invariant term gives rise to the vacuum pressure strong enough to solve the domain wall problem. These possibilities are now under investigation.

In summary, we have shown that the magnetic field is generated spontaneously when the domain wall is present in the early universe. This magnetic field is a candidate of a primodial magnetic field leading to intergalactic or galactic magnetic field in the present universe. We have only discussed the flat domain wall in this paper, but similar results can be obtained even in the case of spherical domain walls. We will present these results in future publications.

The author would like to thank Prof. Y. Hosotani for indicating importance of the zero point energy and valuable comments. He also thank Prof. M. Kawasaki for useful discussions and staff members of theory division in Institute for Nuclear Study, University of Tokyo for their hospitality. 


\section{REFERENCES}

[1] T.W.B. Kibble Phys. Rep. 67 (1980) 183; A. Vilenkin, Phys. Rep. 121 (1985) 263; Kolb and M.S. Turner, "The Early Universe" ( Addison-Wesley, 1990 ).

[2] Ya.B. Zel'dovich, I.Yu. Kobzarev and L.B. Okun, Sov. Phys. JETT 40 (1975) 1.

[3] J. Preskill, S.P. Trivedi, F. Wilczek and M. Wise, Nucl. Phys. B363 (1991) 207.

[4] B. Holdom, Phys. Rev. D28 (1993) 1419; B. Rai and G. Senjanovic, Phys. Rev. D49 (1994) 2729

[5] G. Dvali and G. Senjanovic, Phys. Rev. Lett. 74 (1995) 5178; S.E. Larsson, S. Sarkar and P.L. White, hep-ph/9608319.

[6] C.J. Hogan, Phys. Rev. Lett 51 (1983) 1488; M.S. Turner and L.M. Widrow, Phys. Rev. D30 (1988) 2743; T. Vaschaspati, Phys. Lett. B265 (1991) 258; B. Ratra, Phys. Rev. D45 (1992) 1913; B. Cheng and A.V. Olinto, Phys. Rev. D50 (1994) 2421; M. Gasperini, M. Grovannini and G. Veneziano, Phys. Rev. Lett. 75 (1995) 3796; F.D. Mazzitelli and F.M. Spedalieri, Phys.Rev. D52 (1995) 6694; A. Hosoya and S. Kobayasi, preprint TIT/HEP-298/COSMO-57, 1995.

[7] T. Vaschaspati and A. Vilenkin, Phys. Rev. D30 (1984) 2036.

[8] R. Jakiew and C. Rebbi, Phys. Rev. D13 (1976) 3398; A.T. Niemi and G.W. Semenoff, Phys. Rep. 135 (1986) 100.

[9] Ya.B. Zel'dovich, A.A. Ruzmaikin, and D.D. Sokoloff, "Magnetic Fields in Astrophysics"( Gordon and Breach, New York, 1983 ); E.N. Parker, "Cosmical Magnetic Fields" ( Clarendon, Oxford, 1979 )

[10] S. Deser, R. Jackiw and S. Templeton, Ann. Phys. (NY), 140 (1982) 372; B. Binegar, J. Math. Phys. 23 (1982) 1511.

[11] Y. Hosotani, Phys. Lett B319 (1993) 332; Phys. Rev. D51 (1995) 2022. In these papers 
spontaneous generation of magnetic field in $2+1$ dimensional fermion system has been shown to occur owing to zero point energy of photon.

[12] M.B. Voloshin, hep-ph/9609219

[13] S.A. Abel and P.L. White, hep-ph/9505241 